\section{Case Reports in Ophthalmology}

\begin{tabular}{|c|c|}
\hline \multicolumn{2}{|c|}{ Case Rep Ophthalmol 2014;5:150-156 } \\
\hline $\begin{array}{l}\text { DOI: 10.1159/000363132 } \\
\text { Publisned onine: vvay 21, } 2014\end{array}$ & $\begin{array}{l}\text { (c) } 2014 \text { S. Karger AG, Basel } \\
\text { 1663-2699/14/0052-0150\$39.50/0 } \\
\text { www.karger.com/cop }\end{array}$ \\
\hline
\end{tabular}

This is an Open Access article licensed under the terms of the Creative Commons Attribution-NonCommercial 3.0 Unported license (CC BY-NC) (www.karger.com/OAlicense), applicable to the online version of the article only. Distribution permitted for noncommercial purposes only.

\title{
Bilateral and Simultaneous Central Retinal Vein Occlusion in a Patient with Obstructive Sleep Apnea Syndrome
}

\author{
Andrea Govetto ${ }^{a} \quad$ Ramón Domínguez ${ }^{a} \quad$ Laura Rojas $^{a} \quad$ María Pereiro $^{b}$ \\ Ramón Lorente ${ }^{a}$
}

Departments of aphthalmology and ${ }^{\mathrm{b}} \mathrm{Hematology}$, Ourense University Hospital, Ourense, Spain

\section{Key Words}

Central retinal vein occlusion · Obstructive sleep apnea syndrome $\cdot$ Visual acuity $\cdot$ Optic nerve atrophy

\begin{abstract}
Purpose: To describe a case of bilateral and simultaneous central retinal vein occlusion (RVO) in a young patient diagnosed with obstructive sleep apnea syndrome (OSAS). Case Report: A 38-year-old man with morbid obesity and daytime sleepiness presented with a history of bilateral vision loss. His visual acuity (VA) was hand movements, and fundus examination (FE) revealed bilateral central RVO. General medical examination revealed untreated hypertension and type II respiratory failure. Laboratory tests for thrombophilia showed increased hematocrit (59\%) and high levels of fibrinogen and C-reactive protein. Other causes of congenital and acquired hypercoagulability were ruled out. Pathologic polysomnography led to the diagnosis of OSAS. The patient was treated with antihypertensive drugs and continuous positive air pressure. In addition, he received intravitreal ranibizumab. At 10 months after presentation, his VA was no light perception in the right eye and hand movements in the left eye. FE revealed bilateral retinal and optic nerve atrophy, and the occurrence of a nonarteritic anterior ischemic neuropathy in the right eye was considered.

(C) 2014 S. Karger AG, Basel
\end{abstract}


Govetto et al.: Bilateral and Simultaneous Central Retinal Vein Occlusion in a Patient with Obstructive Sleep Apnea Syndrome

\section{Introduction}

Central retinal vein occlusion (RVO) is a common retinal disorder that usually affects individuals $\geq 55$ of age and, if present in younger individuals, at times hides a procoagulant state $[1,2]$. Central RVO normally occurs unilaterally, but affected patients have an increased risk of developing another vascular occlusion in the fellow eye. On the other hand, bilateral and simultaneous involvement is extremely infrequent.

Recently, RVO has been associated with the obstructive sleep apnea syndrome (OSAS), a condition characterized by repeated episodes of apnea during sleep that increases the risk of cardiovascular disorders [3].

We report the case of bilateral and simultaneous central RVO in a young patient affected by OSAS.

\section{Case Report}

In August 2012, a 38-year-old man came to our center with a 5-day history of progressive and bilateral vision loss. He also reported chronic daytime sleepiness.

His visual acuity (VA) was hand movements in both eyes. A relative afferent pupillary defect (RAPD) was observed in his right eye. Anterior segment examination was unremarkable and intraocular pressure was within normal limits.

Fundus examination (FE) of both eyes revealed vascular congestion and massive preand intraretinal hemorrhages in all quadrants (fig. 1a, fig. 2a). The right eye's optic nerve head, posterior pole and macular region were not evaluable due to hemorrhages, while in the left eye severe papilledema, hard exudates and macular edema were noticed. A diagnosis of bilateral and simultaneous central RVO was formulated. General medical examination revealed morbid obesity with a body mass index (BMI) of 45, untreated hypertension $(170 / 100 \mathrm{~mm} \mathrm{Hg})$ and type II respiratory failure.

The patient was treated with antihypertensive and antiaggregant drugs and referred to the pneumology and hematology department. After 1 week of presentation, the patient underwent fundus fluorescein angiography (FA) (fig. 3a-c, fig. 4a-c). After 1 month of presentation, FE revealed partial resolution of hemorrhages and persistence of macular edema (fig. 1b, fig. 2b). The patient underwent polysomnography which revealed a pathologic apnea-hypopnea index that led to the diagnosis of OSAS, and treatment with continuous positive air pressure (CPAP) was started.

Laboratory tests for thrombophilia showed increased hematocrit (59\%) and high levels of fibrinogen and C-reactive protein (CRP). Other causes of congenital and acquired hypercoagulability like factor V Leiden, mutation G20210A of prothrombin, deficiency of natural anticoagulants and antiphospholipid syndrome were ruled out. After 3 months of antihypertensive treatment, the patient's blood pressure ranged from 140/90 to 135/80 $\mathrm{mm} \mathrm{Hg}$. At 3 months from presentation, the patient was treated with $0.5 \mathrm{mg}$ of intravitreal ranibizumab (Lucentis ${ }^{\circledR}$, Genentech Inc.). He did not come to the follow-up visits, but presented again at 10 months from presentation, complaining of poor vision.

VA was no light perception in his right eye and hand movements in the left eye. FE and FA documented bilateral retinal atrophy (fig. 1c, fig. 2c, fig. 3d-f, fig. 4d-f). Spectral domain optic coherence tomography (SD-OCT) confirmed bilateral macular atrophy (fig. 5a, b). Small remnants of intraretinal fluid not involving the fovea were observed in the right eye (fig. 5a). Due to these findings, the occurrence of a nonarteritic anterior ischemic optic neuropathy (NAION) in the right eye was considered. 
Govetto et al.: Bilateral and Simultaneous Central Retinal Vein Occlusion in a Patient with Obstructive Sleep Apnea Syndrome

\section{Discussion}

To our knowledge, this is the second reported case of bilateral and simultaneous central RVO in a patient affected by OSAS [4]. Recently published studies found a high prevalence of RVO in patients affected by sleep apneas $[5,6]$. These findings can be explained by a broad spectrum of metabolic and hemodynamic changes promoted by OSAS that can lead to a procoagulant state, facilitating thrombus formation and retinal vascular occlusion.

Indeed, OSAS can promote the development of all three factors of the so-called Virchow's triad (hypercoagulability, hemodynamic changes, endothelial dysfunction), and its association with all-cause mortality and cardiovascular disease is well-recognized [7].

Our case was diagnosed with type II respiratory failure, characterized by hypoxemia and hypercapnia, which is characteristic of sleep apnea. Cycles of apnea and subsequent intermittent hypoxemia and hypercapnia can result in cerebral vasodilatation and surges in arterial blood pressure. The cerebral vascular system of OSAS patients is more sensible to arterial pressure changes due to defective mechanisms of autoregulation. Rise in intracranial pressure may result in papilledema and high venous pressure on the optic nerve head [8].

In addition, as the central retinal artery and vein share a common adventitial sheath, vasodilatation of the central retinal artery due to hypercapnia may compress the adjacent vein contributing to its occlusion.

Our patient also presented high hematocrit. Chronic hypoxemia causes an increase of hematocrit and, as a consequence, of blood viscosity to compensate for a lower arterial oxygen tension. Higher blood viscosity can lead to stasis and promote thrombus formation. OSAS also promotes an inflammatory state and, as a result, the level of acute-phase proteins is increased $[9,10]$. In our patient, chronic high serum levels of fibrinogen and CRP were reported.

Fibrinogen is an independent risk factor for cardiovascular disease and an important mediator in the coagulation process, as it can promote platelet aggregation and formation of fibrin clots. CRP is an acute-phase protein and does not only mediate inflammation, but also plays an active role in the coagulation cascade and can be considered an independent risk factor for thrombotic events. CRP can decrease the activity of tissue plasminogen by the activation of plasminogen activator inhibitor- 1 with a subsequent decrease of the fibrinolytic activity. Platelet activity can also be stimulated by cathecolamines, which have increased levels in sleep apnea due to an enhanced sympathetic activation in response to the nocturnal desaturation of oxyhemoglobin [11].

In patients affected by OSAS, other metabolic and biochemical alterations have been found. For instance, an increase in concentration of clotting factors (XIIa and VIIa) was described in two clinical trials [12]. However, such alterations were not found in our case. Furthermore, OSAS is strongly related with systemic hypertension, a major risk factor for RVO. A large community-based study demonstrated that patients with OSAS have a two-fold increase in odds ratio for presenting hypertension [13]. At presentation, our patient had uncontrolled hypertension which may have contributed to the development of central RVO. Finally, at 10 months after presentation, our patient presented important optic nerve atrophy in his right eye, along with a VA of no light perception. Such findings are possible but infrequent in central RVO and, considering the RAPD observed at presentation, we cannot discard that a NAION could have occurred in the right eye.

In fact, NAION has been recently associated with OSAS [14-16]. A cross-sectional study by Mojon et al. [14] found a significantly higher prevalence of OSAS in patients affected by NAION (71\%) compared to matched controls (18\%). Later, a prospective study by Palombi et al. [15] confirmed this finding and reported a relative risk for NAION patients to have a 
Govetto et al.: Bilateral and Simultaneous Central Retinal Vein Occlusion in a Patient with Obstructive Sleep Apnea Syndrome

OSAS of 4.9 compared to the general population, suggesting a clinically significant association between the two conditions.

The pathophysiologic mechanism of NAION in OSAS patients is still unclear. However, direct damage of the optic nerve due to OSAS-induced hypoxia, altered vascular autoregulation and vascular compression may contribute to the development of this condition $[14,15$, $17,18]$.

In our case, an early diagnosis of NAION was not formulated. A RAPD is a common finding in central RVO [19], and the evaluation of the optic nerve head was not possible due to massive retinal hemorrhages. Color vision assessment and peripheral visual field test, recommended in the evaluation of central RVO and NAION, were programmed but not carried out due to very poor patient compliance.

Our patient was treated with CPAP, which can reduce the deleterious effects of intermittent hypoxemia during sleep and improve levels of inflammatory markers, and antihypertensive drugs [10]. On the other hand, no effective medical treatment is established to prevent or treat RVO, and the role of CPAP in preventing NAION is controversial $[16,20]$. Therefore, identifying and treating any underlying condition is important in order to reduce the risk of RVO and, as the consequences of OSAS are systemic, it is important to remark that a multidisciplinary approach is required for optimal management of these patients.

The evolution of the bilateral and simultaneous central RVO in our case was unfavorable, despite all our efforts. Considering that the consequences of central RVO, especially if bilateral, can be very invalidating, it is critical to identify persons who are at higher risk.

Until recently, OSAS was not associated with RVO and few studies on this issue have been published [3-6]. In addition, with the exception of a cohort study by Chou et al. [3], well-designed epidemiological studies investigating whether RVO is more frequent in OSAS patients are lacking. Nevertheless, despite the scarce scientific evidence, in our opinion, ophthalmologists should consider OSAS patients at high risk for RVO. Considering that OSAS can promote a procoagulant state, affected patients may develop RVO more frequently than the normal population. Further well-designed epidemiological studies are required to clarify this issue.

\section{Disclosure Statement}

The authors declare that there are no conflicts of interest.

\section{References}

1 Lahey JM, Tunç M, Kearney J, Modlinski B, Koo H, Johnson RN, Tanaka S: Laboratory evaluation of hypercoagulable states in patients with central retinal vein occlusion who are less than 56 years of age. Ophthalmology 2002;1:126-131.

-2 Kuhli-Hattenbach C, Scharrer I, Lüchtenberg M, Hattenbach LO: Coagulation disorders and the risk of retinal vein occlusion. Thromb Haemost 2010;103:299-305.

-3 Chou KT, Huang CC, Tsai DC, Chen YM, Perng DW, Shiao GM, Lee YC, Leu HB: Sleep apnea and risk of retinal vein occlusion: a nationwide population-based study of Taiwanese. Am J Ophthalmol 2012;154:200-205.

-4 Turati M, Velez-Montoya R, Gonzalez-Mijares CC, Perez-Montesinos A, Quiroz-Mercado H, Garcia-Aguirre G: Bilateral central retinal vein occlusion associated with obesity-hypoventilation syndrome (Pickwickian syndrome). Retin Cases Brief Rep 2009;3:140-143.

$>5$ Glacet-Bernard A, Leroux les Jardins G, Lasry S, Coscas G, Soubrane G, Souied E, Housset B: Obstructive sleep apnea among patients with retinal vein occlusion. Arch Ophthalmol 2010;128:1533-1538.

-6 Kanai H, Shiba T, Hori Y, Saishin Y, Maeno T, Takahashi M: Prevalence of sleep-disordered breathing in patients with retinal vein occlusion. Nihon Ganka Gakkai Zasshi 2012;116:81-85.

7 Sibyl S, Collop N: Latest advances in sleep medicine: obstructive sleep apnea. Chest 2012;142:1645-1651. 
Govetto et al.: Bilateral and Simultaneous Central Retinal Vein Occlusion in a Patient with Obstructive Sleep Apnea Syndrome

8 Lee AG, Golnik K, Kardon R, Wall M, Eggenberger E, Yedavally S: Sleep apnea and intracranial hypertension in men. Ophthalmology 2002;1093:482-485.

-9 Lui MM, Lam JC, Mak HK, Xu A, Ooi C, Lam DC, Mak JC, Khong PL, Ip MS: C-reactive protein is associated with obstructive sleep apnea independent of visceral obesity. Chest 2009;135:950-956.

10 Baessler A, Nadeem R, Harvey M, Madbouly E, Younus A, Sajid H, Naseem J, Asif A, Bawaadam H: Treatment for sleep apnea by continuous positive airway pressure improves levels of inflammatory markers - a metaanalysis. J Inflamm (Lond) 2013;10:13.

-11 Olson LJ, Olson EJ, Somers VK: Obstructive sleep apnea and platelet activation: another potential link between sleep-disordered breathing and cardiovascular disease. Chest 2004;126:339-341.

12 Robinson GV, Pepperell JC, Segal HC, Davies RJ, Stradling JR: Circulating cardiovascular risk factors in obstructive sleep apnoea: data from randomised controlled trials. Thorax 2004;59:777-782.

13 Nieto FJ, Young TB, Lind BK, Shahar E, Samet JM, Redline S, D’Agostino RB, Newman AB, Lebowitz MD, Pickering TG: Association of sleep-disordered breathing, sleep apnea, and hypertension in a large community-based study. Sleep Heart Health Study. JAMA 2000;283:1829-1836.

14 Mojon, DS, Hedges TR III, Ehrenberg B, Karam ZE, Goldblum D, Abou-Chebl A, Gugger M, Mathis J: Association between sleep apnea syndrome and nonarteritic anterior ischemic optic neuropathy. Arch Ophthalmol 2002;120:601-605.

15 Palombi K, Renard E, Levy P, Chiquet C, Deschaux CH, Romanet JP, Pépin JL: Non-arteritic anterior ischemic optic neuropathy is nearly systematically associated with obstructive sleep apnea. Br J Ophthalmol 2006;90:879-882.

16 Stein JD, Kim DS, Mundy KM, Talwar N, Nan B, Chervin RD, Musch DC: The association between glaucomatous and other causes of optic neuropathy and sleep apnea. Am J Ophthalmol 2011;152:989-999.

17 Dhillon S, Shapiro CM, Flanagan J: Sleep-disordered breathing and effects on ocular health. Can J Ophthalmol 2007;42:238-243.

-18 Riva CE, Hero M, Titze P, Petrig B: Autoregulation of human optic nerve head blood flow in response to acute changes in ocular perfusion pressure. Arch Clin Exp Ophthalmol 1997;235:618-626.

19 Servais GE, Thompson HS, Hayreh SS: Relative afferent pupillary defect in central retinal vein occlusion. Ophthalmology 1986;93:301-303.

20 Behbehani R, Matthews MK, Sergott RC, Savino PJ: Nonarteritic anterior ischemic optic neuropathy in patients with sleep apnea while being treated with continuous positive airway pressure. Am J Ophthalmol 2005;139:518-521.

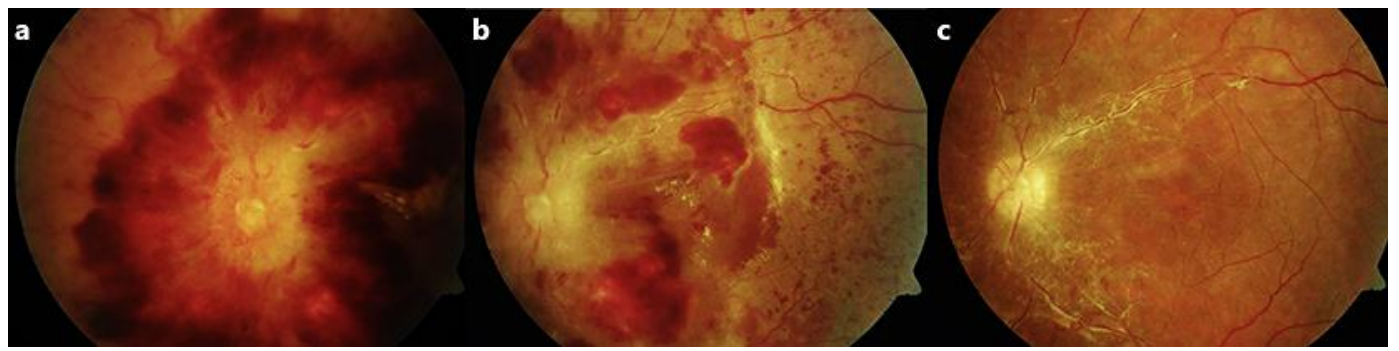

Fig. 1. Fundus photographs of the left eye. a At presentation, central RVO with optic nerve head swelling, extensive intra- and preretinal hemorrhages in all four quadrants are shown. The macular region is not evaluable. Tortuosity and dilatation of retinal veins are seen. b At 1 month, partial resolution of the hemorrhages are seen. The macular region is visible, showing edema and extensive hard exudation. Retinal venous tortuosity and dilatation are still present. c At 10 months, retinal hemorrhages and macular edema resolved. Optic disc atrophy is seen. 
Govetto et al.: Bilateral and Simultaneous Central Retinal Vein Occlusion in a Patient with Obstructive Sleep Apnea Syndrome

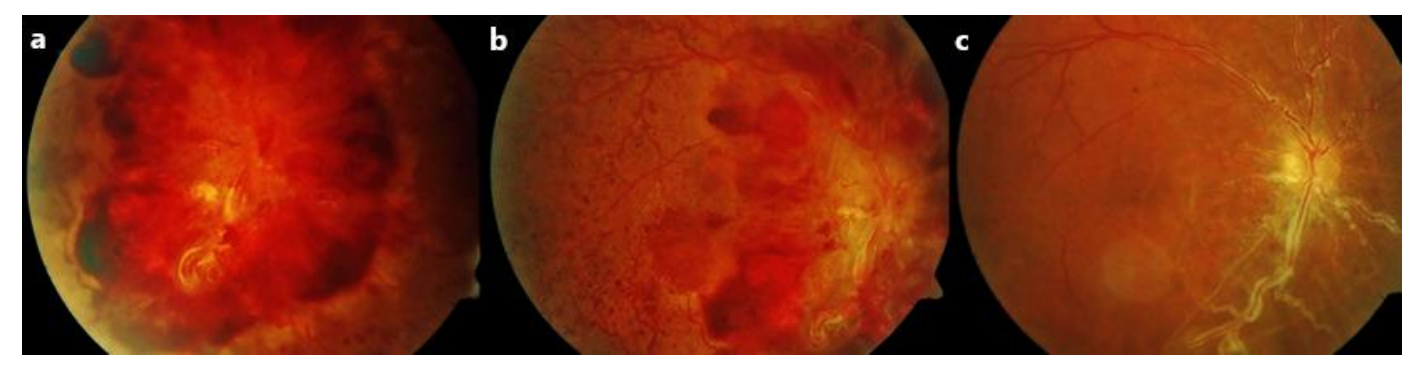

Fig. 2. Fundus photographs of the right eye. a At presentation, central RVO with extensive hemorrhages that limit the evaluation of the optic disc head is shown. The macular region is not evaluable. b At 1 month, optic disk swelling is visible. Retinal hemorrhages limit evaluation of the macular region, where a hard exudation is seen. Extensive retinal vascular tortuosity and dilatation is present. Temporally to the optic disc, head collateral vessels can be seen. c At 10 months, retinal hemorrhages and macular edema resolved. Important optic atrophy is noticed.

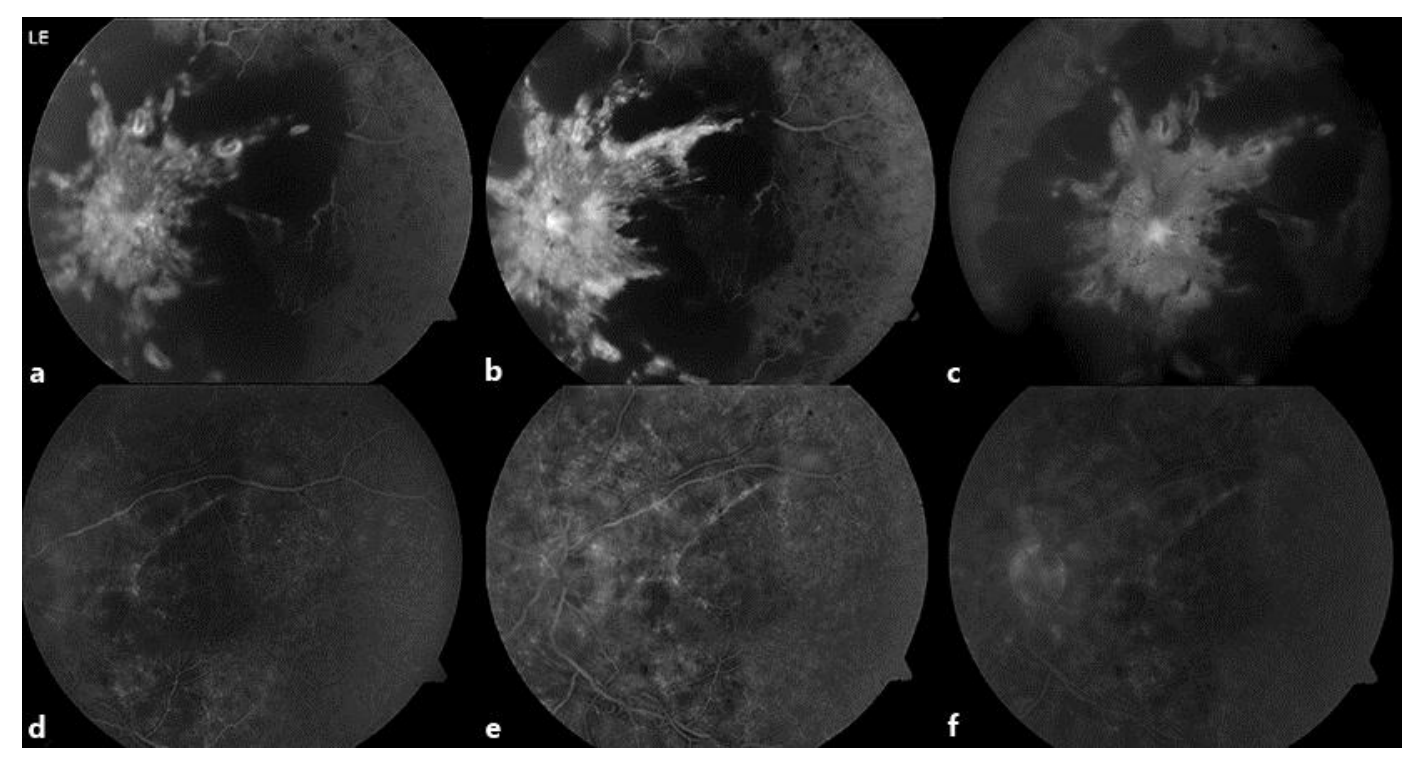

Fig. 3. FA of the left eye. a, b At 1 week, early venous phase (a) and mid phase (b): hyperfluorescence of the optic disc with indistinct borders. Extensive hypofluorescent blockage due to retinal hemorrhages limits evaluation of the perfusion status. Macular edema is noticed. No signs of ischemia are observed. c At 1 week, late phase: hyperfluorescence of the optic disc persists. Extensive hypofluorescent blockage. $\mathbf{d}-\mathbf{f}$ At 10 months, early venous phase (d), mid (e) and late phase (f): no macular edema is seen. Hyperfluorescence due to staining and retinal atrophy are noticed. In the macular region, areas of hypo- and hyperfluorescence due to chronic changes in retinal pigmented epithelium are seen. In the macular and optic nerve regions, neither retinal ischemia nor new vessels are observed. 


\section{Case Reports in \\ Ophthalmology}

\begin{tabular}{l|l}
\hline Case Rep Ophthalmol 2014;5:150-156 \\
\hline DOI: 10.1159/000363132 & $\begin{array}{l}\text { ○ 2014 S. Karger AG, Basel } \\
\text { www.karger.com/cop }\end{array}$ \\
\hline
\end{tabular}

Govetto et al.: Bilateral and Simultaneous Central Retinal Vein Occlusion in a Patient with Obstructive Sleep Apnea Syndrome

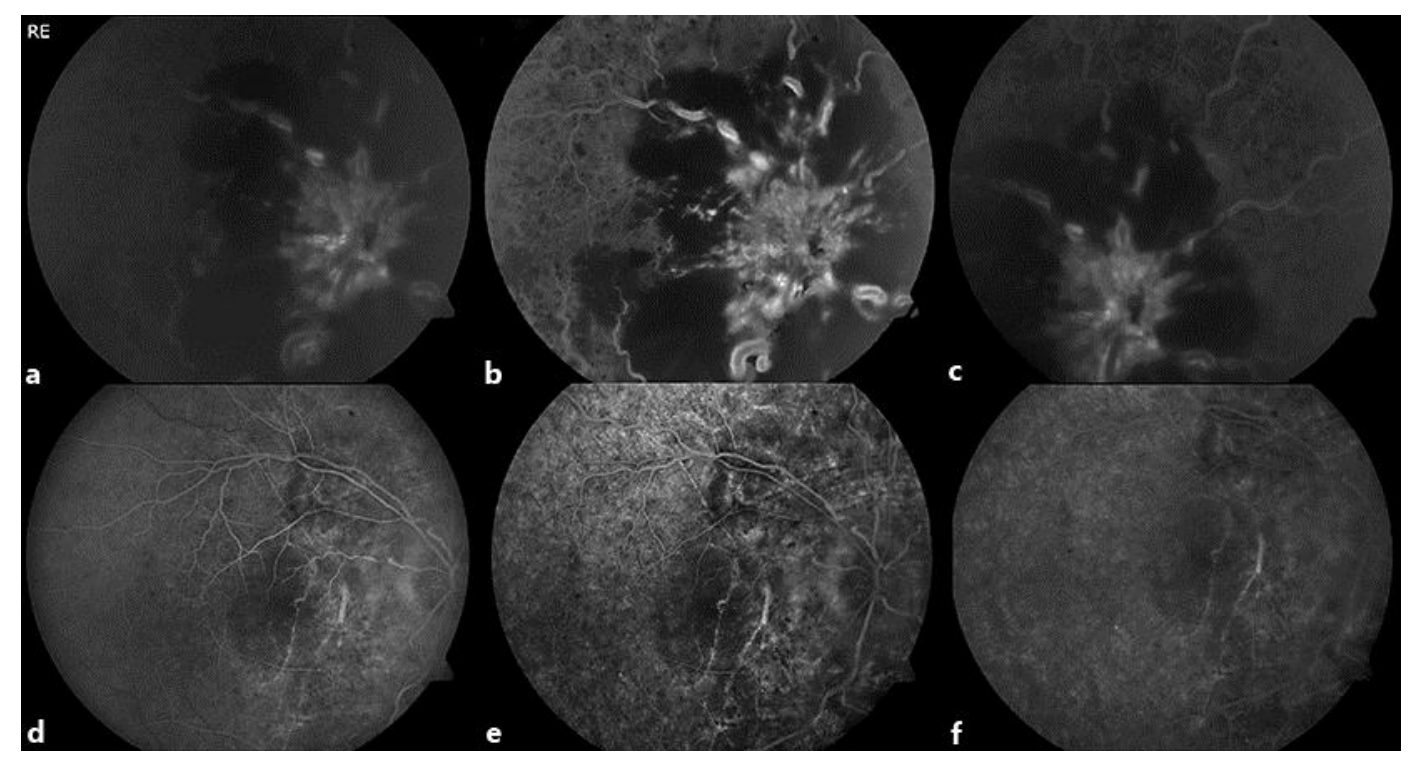

Fig. 4. FA of the right eye. a, b At 1 week, early venous phase (a) and mid phase (b): important blockage as a result to retinal hemorrhages limits evaluation of the posterior pole. Optic disk head swelling is seen. Important vascular tortuosity and dilatation is noticed. The macular region and the optic disc are not fully evaluable. c At 1 week, late phase: optic disc swelling persists as well as blockage due to hemorrhages. $\mathbf{d}-\mathbf{f}$ At 10 months, early venous (d), mid (e) and late phase (f): areas of hypo- and hyperfluorescence due to chronic changes in the retinal pigmented epithelium and retinal atrophy are seen. In the macular and optic nerve regions, neither retinal ischemia nor new vessels are observed.

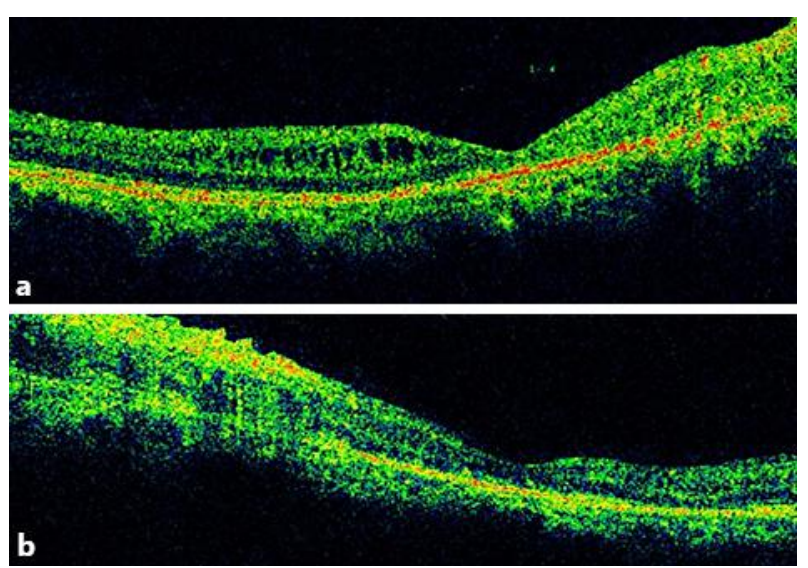

Fig. 5. SD-OCT of the right and left eye. a SD-OCT of the right eye at 10 months from presentation: important macular atrophy can be seen. Small remnants of intraretinal fluid not involving the fovea are seen. $\mathbf{b}$ SD-OCT of the left eye at 10 months from presentation: similarly to the right eye, macular atrophy is seen. No intra- or subretinal fluid is noticed. 